\title{
Towards a Collective Knowledge for a Smart Electric Vehicle Charging Strategy
}

\author{
João C. Ferreira \\ GuIAA - DEETC - ISEL \\ Lisbon, Portugal \\ jferreira@deetc.isel.ipl.pt
}

\begin{abstract}
In this work is proposed the design of a system to create and handle Electric Vehicles (EV) charging procedures, based on intelligent process. Due to the electrical power distribution network limitation and absence of smart meter devices, Electric Vehicles charging should be performed in a balanced way, taking into account past experience (spread in a social network). In order to allow information exchange and to help user mobility, it was also created a mobile application to assist the EV driver on these processes. This proposed Smart Electric Vehicle Charging System uses Vehicle-to-Grid (V2G) technology, in order to connect Electric Vehicles and also renewable energy sources to Smart Grids (SG). This system also explores the new paradigm of Electrical Markets (EM), with deregulation of electricity production and use, in order to obtain the best conditions for commercializing electrical energy.
\end{abstract}

Keywords; Collective Knowledge; Data Mining; Simulation; Electric Vehicle; Energy Market; Smart Grids; Vehicle-to-Grid; Mobile Device

\section{INTRODUCTION}

New paradigms are emerging, like the Electric Vehicle (EV), the Smart Grids (SG), the Vehicle-to-Grid (V2G), and the Electrical Markets (EM). EM is the consequence of the deregulation of electricity production and use, where power suppliers and consumers are free to negotiate the terms of their contracts. Also EVs integration on current electrical distribution network, without violating the system's technical restrictions, requires electrical data consumption analysis and smart charging approaches, where EV batteries charging or discharging processes need to be coordinated among the several users. In this complex scenario, information knowledge related with charging periods, prices, decision of charging or discharging EV batteries, needs the assistance from data mining processes and the establish of a collective knowledge. Several issues have to be considered and analyzed before taking action. Although enormous data volumes related with this processes are stored day by day, and hour by hour, it is impossible (through human analysis or with traditional technology) to obtain knowledge from this data, in order to take wise decisions. This topic already catches a lot of attention of the scientific community under the topic of EM $[1,2,3]$, and in this paper it is proposed a similar approach based on social networks for the charging or discharging process of the batteries in an EV. This new reality brings additional problems, such as: (1) overload of electrical energy distribution network, if there is a considerable amount of EVs charging at the same time; (2) home consumption and contractual power limitation; (3)

\author{
João Luiz Afonso \\ DEI - Universidade Minho \\ Guimarães, Portugal \\ jla@dei.uminho.pt
}

buying electricity at lower prices when renewable energy is produced in excess, and selling electricity at higher prices when the demand for energy is superior to the offer in the electrical network. Smart Grids with measuring devices and a communication infrastructure, among other devices, is part of a solution for this problem in a near future, and therefore, the proposal here presented could facilitate the creation of SG (because it is possible to perform some functions related with the control of power available in the electrical network based on historical data), and later, the proposed system, integrated on a SG, also could execute tasks related with EM (finding the hours with best prices for charging or discharging the EV batteries, and other home personalized functions).

In this paper is proposed a central system, where a central information repository can store and manage historical data on electricity consumption and production. From this central repository it is possible the development of tools to extract knowledge from past electricity exchange log files, EM prices, renewable energy availability, home energy consumption (if EV is connected at home) and electrical distribution network constraints. Also, the social networks are integrated as a tool to share and spread useful related information. This central repository will be later, in a Smart Grid environment, a fundamental module to store all kind of SG data and to solve the problems of different data format diversity.

Our research proposal focuses on the manipulation and retrieval of digital data based on a collaboration environment. The goal is to move towards the creation of a collective knowledge for the automatic diffusion of user/community relevant information supported on a mobility system applied to EV charging problem. Our research approach has 3 main working guidelines. The bottom line is the semantic information repository that aggregates digital data and semantic descriptors which are created through annotation within a collaborative oriented environment. The intermediate line is the stakeholders' data manipulation that includes the definition of user profiles that are clustered into a community. The top line is the selective dissemination of knowledge, which includes the filtering of user-relevant information and the inference of implicitly represented knowledge. The notion of collective knowledge, depicted in Figure 2, emerges from the systematic and detailed characterization of the above 3 lines of research. 


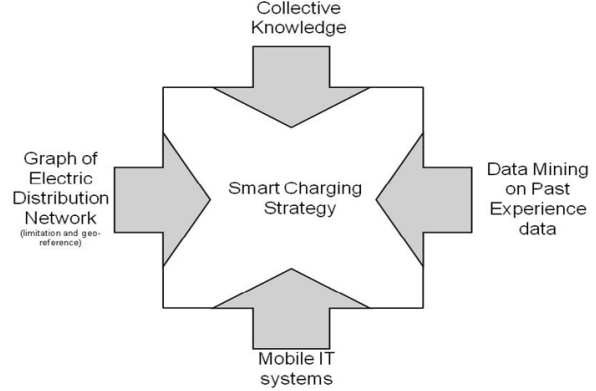

Figure 1. Main approaches towards a smart charging strategy for EV

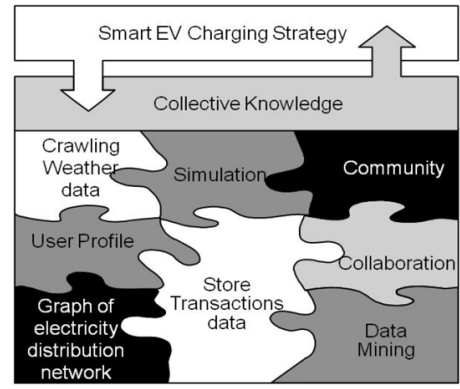

Figure 2. Main approach towards a collective knowledge

\section{SOCIAL NETWORKS AND COLLECTIVE KNOWLEDGE}

The Social Web represents a set of web sites and applications in which user participation is the primary driver of value. The principal of such systems is well described by Tim O'Reilly [4] and this development is around Web 2.0 [5] principals. Examples of these types of initiatives are Wikipedia, MySpace, YouTube, Flickr, Del.icio.us, Facebook, and Technorati. Discussions of the Social Web often uses the phrase "collective intelligence" or "wisdom of crowds" to refer to the value created by the collective contributions of all these people writing articles for Wikipedia, sharing tagged photos on Flickr, sharing bookmarks on del.icio.us. Knowledge sharing have great potential due to the diversity of information available, diversity of individual cultures and the low costs of gathering and computing over big network (Internet). Currently we have millions of persons offering their knowledge online, which means that the information is stored, searchable, and easily shared, so the big challenge is to find the right match between what is put online and the approach to take the right/useful information from this data. A collective intelligence/knowledge can result from the data collected from all those people are aggregated and transformed to create new knowledge. This collaborative approach had the spam problem and other fraudulent sources in the mix, simply collecting the contributions of the masses does not lead to new levels of intelligence, but by the measurement of user contributions we can reduce the impact of this problem.

A synergy can be established between people, which are the producers, and the consumers of information and machines in the role of enablers (store, search, combine, inference). In the communication process people create knowledge and machines can help on this process, giving tools to help the process of communication and information sharing. So our approach is based on the knowledge offered by millions of people (experts and also novice users) with a background infrastructure for storing, searching and easily sharing of information. There has been an increasing interest in developing Web applications that include a social component. For example, the primary emphasis of sites such as Facebook and LinkedIn is in allowing people to express the connections in their social networks, forge new connections and engage in social interactions online.

In slight contrast, social annotation and bookmarking services, such as those summarized by Hammond [6], allow individuals to store and annotate items for their own usage, but also share these resources with others through the social networking aspects of the sites. One form of this collaboration is Word-of-mouth recommendation and referrals from others. These are powerful mechanisms for helping people acquire information and solve problems, in domains as diverse as finding piano teachers [7] and successfully completing projects in the workplace [8]. Social networks of known individuals can serve as both a source of new information and as a filter to identify the information or items most relevant to one's specific needs $[9,10]$. These processes have been extensively studied in a number of disciplines, particularly sociology, psychology, marketing and organisational sciences. In one of the earlier studies on the subject [11], provides an account of how interpersonal communication networks in local neighbourhoods can influence purchasing behaviour of domestic appliances. This study emphasised the existence of social networks that, through their role in information flow, can account for the non-random distribution of consumption patterns within the wider population.

\section{SMART EV CHARgING SYSTEM}

Our investigation proposal is to bring computer science work on software development, Web 2.0, geographic information systems, mobile computation and wireless communication, to a new growing area of Smart Grids (SG) and EV (Electric Vehicle). Due to the increasing complexity and diversity of options, users in Electrical Markets (EM), when performing an EV charging process, will need the help from software application, mainly to mobile devices. So our proposals, presented in this paper, are the conception and the creation of a mobile application and surrounding system, to help users on EV charging or discharging process, and also on EM participation. The main modules of the proposed system, called Smart EV Charging System, and which is illustrated on Figure 3, are: (1) Central Repository, with information about user energy consumption (amount and time), energy production with available information of power, energy supplier and source (e.g., hydropower, wind power, photovoltaic, etc), energy prices and weather information (temperature, wind direction and speed, rain amount, solar radiation, etc), user profile information; (2) Web Relevant information through a specialized crawler. We implemented 
a Weather crawler, based on a web robot to pick weather information from pre-defined sites; (3) Geo-reference graph based on electricity distribution network; (4) Simulation Tool, based on Netlog; (5) Community creation based on clustering available user profile; (6) data mining approach for consumption data analyses; and (7) Mobile Application, an application to run on a mobile device (like PDA or IPphone) to receive and send control information for charging the EV batteries. These modules are described in the next sections. Main output information is: (1) user personalized information related to $\mathrm{V} 2 \mathrm{G}$ charging process; (2) Community relevant information; and (3) smart charging strategy taking in account prices and distribution limitations.

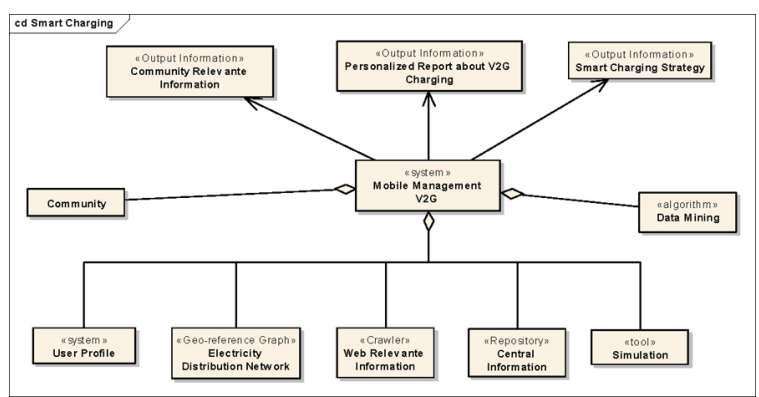

Figure 3. Main modules of the Smart EV Charging System

\section{HOME CONSUMPTiON SimUlation AND GEO- REFERENCE ELECTRICITY DISTRIBUTION NETWORK GRAPH}

An agent based simulation attempts to simulate an abstract model of a particular system. Computer simulations have become an useful part of mathematical modeling of many natural systems in physics, chemistry and biology, and of human systems in economics, psychology, social science, and engineering. Simulations can be used to explore and gain new insights into new technology, and to estimate the performance of systems too complex for analytical solutions. This approach already has been applied for EM [12, 13, 14], creating a simulation environment for market prices determination based on consumers demand, and for production capacity of producers. Our main idea is to simulate consumers consumption taking also into account unexpected user behavior, using past experience (consumption log files), and then, represent the information in an electrical network distribution graph. There are several tools that can be used for this purpose, from which NetLogo tool has been chosen. NetLogo is a free agent-based simulation environment that uses a modified version of the Logo programming language, providing a graphical environment to create programs that control graphic "turtles" that reside in a world of "patches," which is monitored by an "observer". NetLogo also includes an innovative feature called HubNet, which allows groups of people to interactively engage in simulation runs alongside of computational agents. The area with the distribution of the electrical network is manually transformed in a graph, where we add geographic information and power limitation between the nodes. This is a slow process where we expect in a near future to introduce some automation. Assumptions on consumer's behavior are considered: (1) Consumers define their house and family (number of house divisions, number of persons); (2) They define the number and type of electrical appliances from a pre-defined list; and (3) They define also their usual routine (arrival time, departure time). Tracking system data can tune arrival and departure times. Every consumer has its own behavior and changes or unexpected behavior are randomly generated at the beginning of the experiment, using an array of integers. Each consumer is represented by an agent who knows contractual power limitation and also the distribution.

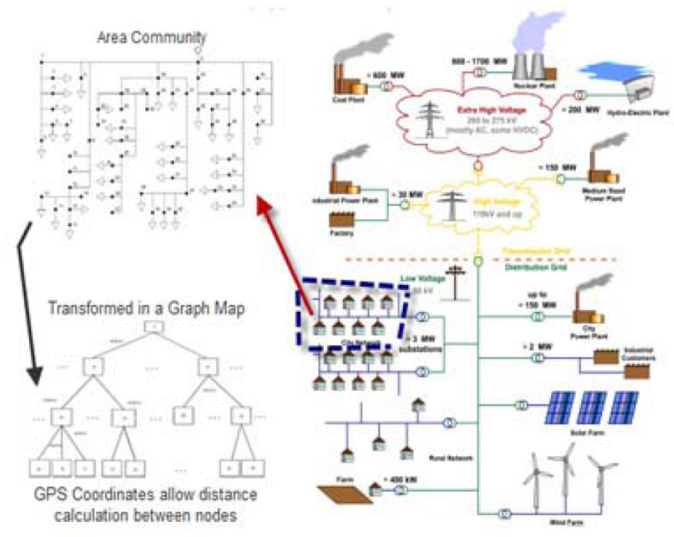

Figure 4. Electrical network distribution, transformed in a graph.

On the simulation tool (Netlog) we follow a bottom-up approach where we estimate consumption based on consumer profile and historical consumption data. Weather information (temperature) is used as a percentage increase factor on usual consumption. Each consumer is represented by an agent that is based on historical data, profile and temperature information, based on a random function for energy consumption, which is estimated at every 15 minutes (this time interval is configurable). Each agent has a utility function, but the agent is not optimizing it because this process is too expensive under many aspects: in terms of information retrieval cost, in terms of information processing costs from a computational point of view, and in terms of cognitive effort in searching alternatives. We decide to model each consumer as a node on a network distribution graph. Simulation takes into account house power limitation contract and electrical energy distribution limitations. Main Output information is the visualization of electrical distribution network on a graph with the indication of power limits. Red color means above power capacity, which indicates that $\mathrm{EV}$ charging should be processed in intelligent interactive process; green color means that we can charge EV batteries on full power. We can estimate consumption and use this information for a smart EV charging, without measuring devices and real time information. The main application screen of the consumption simulation is illustrated on Figure 5.

Relatively to Electrical Market (EM) functions, we can aggregate energy production and consumption data, and 
based on this simulation estimate prices and then determinate the best charging or discharging periods.

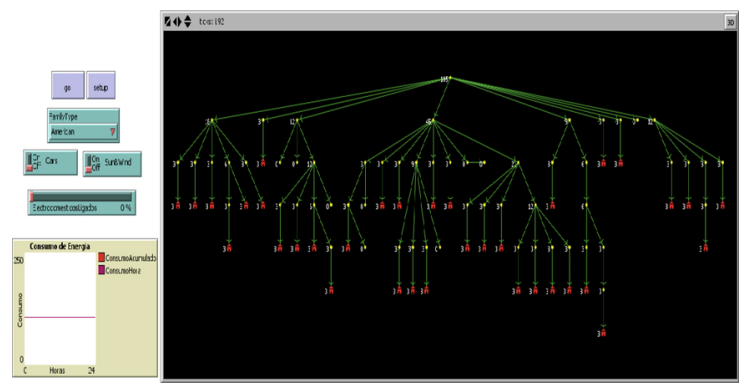

Figure 5. Simulation application on Netlog.

\section{MOBILE V2G MANAGEMENT SYSTEM}

Vehicle-to-Grid (V2G) technology utilizes the stored energy in Electric Vehicle (EV) batteries to deliver electricity back to the electrical network, when the network operators request it. Revenue from $\mathrm{V} 2 \mathrm{G}$ will reduce the customer cost of EVs. Besides, V2G technology will also increase the efficiency and reliability of the existing electrical networks, decreasing fossil fuel use, and making possible a much larger share of intermittent renewable energy sources, like wind power and solar photovoltaic.

EV charging will benefit from past data analysis and remote interaction. Mobile applications are a raising business area in Computer Science, with the widespread utilization of small mobile devices (e.g., PDA, IPhones), and the increase of bandwidth and availability of wireless communications. Our proposal is to implement communication devices to take data from charging devices and to send commands to them. This communication is performed from the Charging System to a user home PC or to a remote server. We shall differentiate communication devices and communication protocols to the following cases: (1) Apartments, where distances to home computers or to the local server are considerable (in this case we will use Wimax); and (2) Individual houses, where distance to the home computer is small. The main functionalities of the Mobile application are: (1) Registration: registration page for new users; (2) Password Recover: form for password recovery; (3) Login: home page of the application - the user is redirected to this page after login; (4) Profile Creation: page created for user profile by entering information on the EV; (5) Personalized Charge Profile: page load profiling, through the introduction of information regarding the date / time of travel, number of $\mathrm{Km}$ you intend to accomplish, and minimum SOC (State of Charge) allowed for the EV batteries; (6) Statistics: home energy consumptions, weekly, monthly and annual energy expenses, price variation of electricity, charging periods, among others. The present application on the server is subdivided into five main modules: (1) Interpreter of Downloaded Files - this module will be responsible for reading and interpreting the files loading, giving the system a layer of abstraction over the file format of text issued by the loading system; (2) Smart Grid Interface - this module will be responsible for the interaction with the electrical network, i.e., it controls the flow of energy from or to the electrical network, with the objectives of helping network stability, and also, managing information on the variation of electricity prices, to optimize the profits obtained with the selling of energy to the electrical network; (3) User Manager - module responsible for registering the users and their EVs, allowing the recording and editing of users data, as well as the removal of users (if defined rules are not accomplished by specific users) - this module is also responsible for verification of user identity and ownership of registered vehicles (through the transmission of data received from the user to the authorities), and for performing regular cleaning from the database of users categorized as "spam"; (4) Manager Profiles - a user can set one or more load profiles for each of the vehicles registered by him. A common practice is, for example, the definition of profiles and needs of different charging to be carried out during the week (weekdays) over the weekend; and (5) Manager Central consists in the main module of the V2G Smart System, interacting with various modules mentioned above, and managing the distribution of system information (from other modules and database).

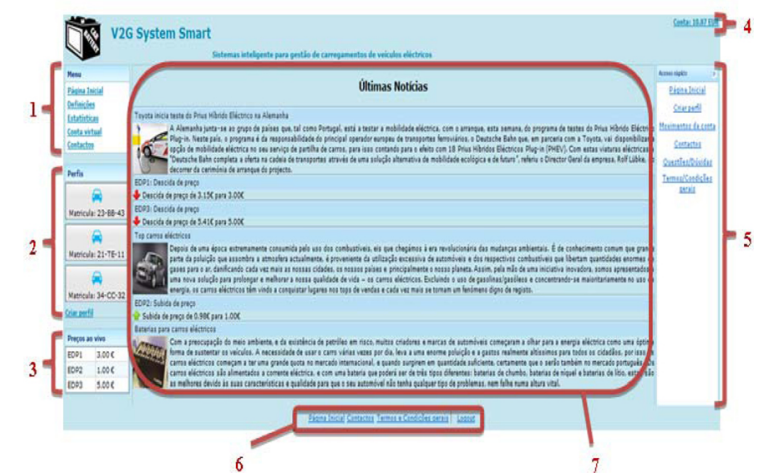

Figure 6. V2G Smart System Mobile Application: (1) Main menu with information of user definition, statistics, and virtual account; (2) User profile; (3) EM prices - simulated with base on renewable energy production, based on installed capacity and weather information (wind direction and speed, rain percentage, temperature, solar radiation); (4) User account related with the EM; (5) Personalized menu links for user's fast access; (6) Application default menus; (7) EV and EM related news, created from personalized web search robot.

In relation to technologies to be used in the development of these applications, it was decided that, the management system database, using MySQL Server, will be weighting the choice that it is open source. The development of web application will be held in Java, and all graphical development will be carried out using the ZK Framework, which facilitates the development of Web applications with Ajax, and has the advantage of being open source. The development of the environment, using the Eclipse Project, will be integrated with the ZK plugin, with main modules: (1) ZK Loader - consists of a servlet (server side component that generates HTML and XML data to the presentation layer of a Web application) that processes the requests for resources $\mathrm{zk}$; (2) ZK Client Engine - is processed on the client's browser and is responsible for monitoring the events page and 
realization of their requests to the server by Ajax technique. This engine is generated by $Z K$ Loader when processing HTTP requests, and is sent in response to the client in the form of JavaScript code; (3) ZK Asynchronous Update another servlet that serves asynchronously requests made by the $Z K$ Client Engine. These requests are caused by interaction of the user with the various components of the page. The $Z K$ Asynchronous processes the events triggered by the user, sending the response to the $Z K$ Client Engine, encoded according to a protocol's own framework, which typically consists of changes to the page content; and (4) Index - home page of the application, from which the user can perform the login in the application or access the registration page if it is your first access.

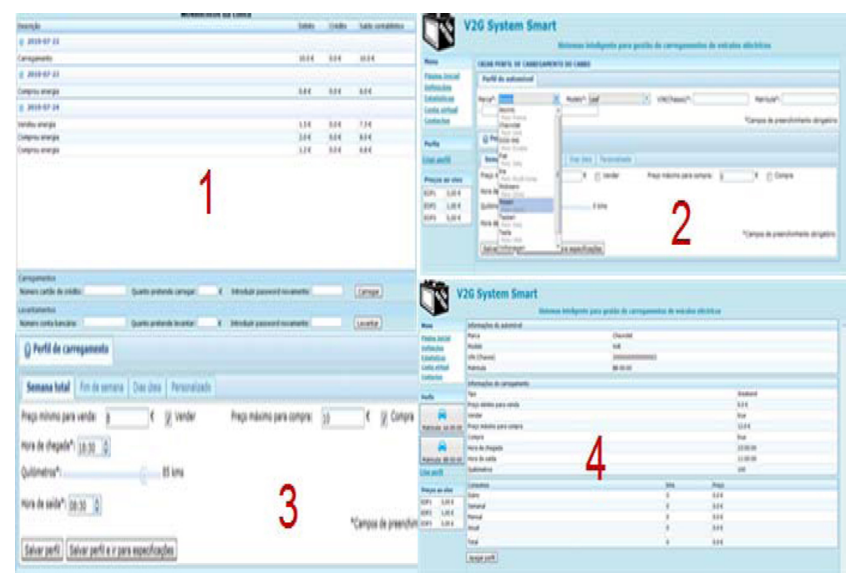

Figure 7. Main application functionalities menu: (1) Tracking of electricity charging and discharging process; (2) Creation of a profile (predefined information, like EV type, helps the user on this process; (3) User profile with the identification of travel periods and electricity prices to sell and buy; (4) Report with all user profile information.

\section{CONCLUSIONS}

This paper describes work that has been developed in order to provide a conceptual system to assist and manage Electrical Vehicles (EV) charging process. This proposed Smart EV Charging System uses Vehicle-to-Grid (V2G) technology, in order to connect not only Electric Vehicles, but also renewable energy sources, to Smart Grids (SG). The new paradigm of Electrical Markets (EM), with deregulation of electricity production and use, is also explored in this developed system, in order to optimize the prices of selling or buying electrical energy, to or from the electrical network. In the proposed Smart EV Charging System, the introduction of mobile applications will facilitate connectivity user's interaction. The Central Information Repository, with Data Mining approaches, can be used to program and assist smart EV charging, taking into account the electrical network distribution limitation. A simulation tool helps on this smart charging process, and can be used to identify overloaded electrical distribution lines, and also to simulate behavior and operating conditions under different assumptions. Electrical energy producers can also benefit from this data collection and manipulation, because they can tune their production according to users' consumption needs. The application of Artificial Intelligent approaches has a great potential, once we are able to store consumption and production data and the knowledge information created, which can help both consumers and producers. Mobile devices and applications will help on the access to information.

\section{ACKNOWLEDGEMENT}

The authors are grateful to the FCT (Fundação para a Ciência e a Tecnologia) and to the MIT-Portugal Program, for funding the Project MIT-PT/EDAM-SMS/0030/2008.

\section{REFERENCES}

[1] C. Farmer, P. Hines, J. Dowds and S. Blumsack, 2010. Modeling the Impact of Increasing PHEV Loads on the Distribution Infrastructure. 43rd Hawaii International Conference on System Sciences 2010

[2] Zhou, Z.; Chan, W.; and Chow, J. 2007. Agent-based simulation of electricity markets: a survey of tools. Artificial. Intelligence Review 28(4):305-342.

[3] A. Ugedo and E. Lobato, 2007. Generator Load Profiles Estimation Using Artificial Intelligence. Intelligent Systems Applications to Power Systems, 2007. ISAP 2007. International Conference on, vol., no., pp.1-6, 5-8 Nov. 2007.

[4] T. O'Reilly. What Is Web 2.0: Design Patterns and Business Models for the Next Generation of Software, September 30, 2005. $<$ http://www.oreillynet.com/pub/a/oreilly/tim/news/2005/09/30/whatis-web-20.html>

[5] Web 2.0 Conferences. http://www.web2summit.com/

[6] Hammond, T., Hannay, T., Lund, B. and Scott, J. (2005) Social Bookmarking Tools (I): A General Review. D-Lib Magazine, 11 (4).

[7] Johnson B. J. and Reingen, P. H. (1987) Social Ties and Word-ofMouth Referral Behaviour. Journal of Consumer Research, 14 (3), pp.350-362.

[8] Cross, R., Rice, R. E. and Parker, A. (2001) Information Seeking in Social Context: Structural Influences and Receipt of Information Benefits. IEEE Transactions of Systems, Man, and Cybernetics, 31 (4), pp.438-448.

[9] Granovetter, M )1983) The Stength of weak Ties: A Network Revisited. Sociological Theory,1,pp.201-233

[10] Kautz, H., Selman, B. and Shah, M. (1997a) The Hidden Web. AI Magazine, 18 (2), pp.27-36.

[11] Whyte, W. H. W. J. (1954) The Web of Word of Mouth. Fortune, November

[12] V. Figueiredo, F. Rodrigues, Z. Vale, J.B. Gouveia. 2010. An Electric Energy Consumer Characterization Framework based on Data Mining Techniques; IEEE Trans. on Power Systems; Vol20, n.2, pp. 596-602.

[13] J.H.Zhao, Z.Y.Dong, X.Li, and K.P.Wong, 2007. A framework for electricity price spike analysis with advanced data mining methods, Power Systems, IEEE Trans. on Issue Date, vol 22, Issue 1, Feb. 2007, pp: $376-385$

[14] B. D. Pitt and D. S. Kitschen, 1999. Application of data mining techniques to load profiling. IEEE International Conference on Power Industry Computer Applications, pp. 131-136, May 1999. 